Para citar este artículo: Aguilar Aguilar, R., García Espinosa, S. \& Guerrero García, H. R. (2021). La trayectoria semántica de la Sustentabilidad. Sostenibilidad: económica, social y ambiental, 3, 63-75. https://doi.org/10.14198/Sostenibilidad2021.3.04

\title{
La trayectoria semántica de la Sustentabilidad
}

\section{The semantic trajectory of Sustainability}

\author{
Rosenda Aguilar Aguilar \\ Universidad Michoacana de San Nicolás de Hidalgo, México \\ rosenda.aguilar@umich.mx \\ https://orcid.org/0000-0003-3392-9062 \\ Salvador García Espinosa \\ Universidad Michoacana de San Nicolás de Hidalgo, México \\ salvador.garcia.espinosa@umich.mx \\ https://orcid.org/0000-0002-7871-5837 \\ Hilda Rosalba Guerrero García-Rojas \\ Universidad Michoacana de San Nicolás de Hidalgo, México \\ hilda.guerrero@umich.mx \\ https://orcid.org/0000-0002-4226-8027
}

\section{RESUMEN}

El manejo cotidiano de los términos sostenible y sustentable en el discurso político, económico y social inició en los años ochenta del siglo XX. Desde ese momento hasta el día de hoy, diversos esfuerzos para alcanzar la sustentabilidad se han diluido en función de las múltiples interpretaciones antrópicas que han surgido alrededor del concepto. Lo anterior se considera un problema, ya que la falta de homogeneidad semántica constituye uno de los factores que han dificultado la concreción acertada de la sustentabilidad. El presente texto utiliza el análisis bibliográfico como principal herramienta metodológica para abordar de forma teórica el tema desde la perspectiva histórica de la polisemia del concepto. El artículo respalda la hipótesis de que, para lograr la sustentabilidad de cualquiera de las esferas antrópicas, es fundamental propiciar primeramente la sustentabilidad del entorno natural con el que todavía contamos.

Palabras clave: sustentabilidad; polisemia; semántica; economía. 


\begin{abstract}
The everyday use of the term sustainable in the political, economic and social discourse began in the eighty's decade of the twentieth century. From that moment to the present day, various efforts to achieve sustainability have been diluted due to the multiple anthropic interpretations that have emerged around the concept. This is considered a problem, since the lack of semantic homogeneity is one of the factors that have hindered the successful realization of sustainability. This text uses bibliographic analysis as the main methodological tool to approach, in a theoretical manner, the subject from the historical perspective of the polysemy of the concept. The article supports the hypothesis that, in order to achieve sustainability in any of the anthropic spheres, it is essential to first promote the sustainability of the natural environment we still have.
\end{abstract}

Keywords: sustainability; polysemy; semantics; economy.

\title{
1. Las decisiones económicas y la Sustentabilidad
}

Desde 1932 se popularizó una definición de la Economía que la refiere como una ciencia que estudia "la conducta humana como una relación entre los fines ilimitados y los medios limitados que requieren una aplicación optativa" (Robbins, 1932). Lo anterior significa que el campo de estudio de la Economía es la conducta humana durante los procesos de, adquisición de recursos de la naturaleza, decisión del uso que les dará, y determinación de cómo los distribuirá para el consumo propio y de otros individuos. El autor de la definición - el economista británico Lionel Robbins - expuso también que esta disciplina está formada por todas las decisiones que los seres humanos tomamos, en un territorio común en un tiempo específico, respecto a cómo utilizar los recursos disponibles para satisfacer a la población. De esta forma, pescar 10 toneladas de sardina para enlatarlas y venderlas en el noroeste de México, representan decisiones económicas.

Como parte de la reflexión del significado de la Economía, es importante resaltar que los seres humanos expresamos constantemente tener necesidades infinitas, pero que los recursos del entorno son finitos (Abbot et al., 2019). Por lo tanto, nunca podrían satisfacerse todos los deseos humanos, ya que, aunque se deseara pescar 100 toneladas de sardina diariamente por todos los años de existencia, los recursos en algún momento se agotarían.

Bajo el marco anterior, resulta interesante explorar la forma en que se han tomado las decisiones económicas en nuestro Planeta. De acuerdo con Kraus (2011), podrían existir tres estructuras de decisión. En la primera es el Estado quien guía y determina la actividad económica ostentando una amplia influencia en la dirección que toma el uso de recursos - se le conoce como economía de administración estatal-; en la segunda, el Estado solamente participa como elemento negociador en situaciones de crisis y además es el responsable de colocar marcos y límites dentro de los cuales se desarrolla la libertad económica — se trata de la economía social del mercado-; y finalmente, en la tercera, el Estado permanece fuera de la esfera de las decisiones económicas en el más alto grado posible a efecto de que el libre mercado conduzca las decisiones —es el capitalismo laissez-faire- (Kraus, 2011). En la segunda y tercera estructura quienes dirigen el funcionamiento son los productores y consumidores, ambos componentes básicos del mercado.

Al comparar entre sí esas tres estructuras de decisión podrá notarse que existen enormes diferencias en sus implicaciones sociales, políticas y económicas, no obstante, en ellas prevalece algo en común: son las necesidades humanas las que guían las decisiones 
económicas, y en ningún caso han participado de esos acuerdos las necesidades de regeneración, de adaptación, o de limpieza que son necesarias para la atmósfera, la hidrósfera, la biósfera o la litósfera. Más aún, a partir de los años cincuenta del siglo XX, las decisiones económicas que se tomaron en gran parte del mundo solo buscaron acelerar el crecimiento y desarrollo económico e ignoraron por completo que la contraparte de la naturaleza era finita y requería de procesos de restablecimiento y regeneración.

En este sentido, los abusos en el uso y extracción de los recursos naturales, la sobrecarga de desechos en todos los ecosistemas, y los errores en el manejo de la naturaleza, han sido diversos, incrementales y mínimamente evitados. Lo anterior ha sido acompañado por un tipo de ceguera que omite la identificación de daños, pero que con gran agudeza propicia el crecimiento y desarrollo económico en cualquiera de las estructuras de decisión comentadas —administración estatal, social del mercado o del tipo laissez-faire-.

Con la intención de mostrar algunos ejemplos de la actividad económica, se pueden señalar casos regionales como la desaparición del Mar de Aral en Uzbekistán a causa de la producción algodonera en un tiempo tan corto como el de una generación (Williams, 2005), o las cinco islas de basura plástica diseminadas por los océanos Pacífico Norte y Sur, Atlántico Norte y Sur, e Indico, que crean condiciones de peligrosa contaminación global (Avio et al., 2017). Para tener información de más de 3.000 de estos casos que actualmente son conflictos socio-ecosistémicos importantes, se recomienda examinar el Mapa Mundial de Justicia Ambiental (Environmental Justice Organisations, Liabilities and Trade, revisado 2021).

Sin embargo, la expresión más peligrosa de las actividades humanas hoy en día es el Cambio Climático, cuya presencia a escala global representa un problema en curso y de alarmante crecimiento. Se trata de un conflicto socio-ecosistémico originado por la aportación humana de gases de efecto invernadero (GEI) tales como dióxido de carbono, metano, óxidos de nitrógeno, y gases fluorados, que provienen de las siguientes actividades económicas: quema de combustibles en industrias y transportes; ganadería; agricultura por inundación; uso de fertilizantes nitrogenados; empleo de refrigerantes, espumantes y propelentes; e incendios en ecosistemas, como los ocurridos en 2019-2020 en la Amazonia Central de Brasil (Cruz et al., 2020) y en Australia (Yu et al., 2020). Todo lo anterior expone las graves fallas que el ser humano ha cometido durante la toma de decisiones económicas con los recursos planetarios.

Debe subrayarse que, desde los primeros indicios de daños ambientales, hubo profesionistas, investigadores, teóricos de diferentes disciplinas, activistas y políticos que alertaron de las futuras anomalías, perturbaciones y consecuencias que se conseguirían con esas decisiones económicas. Cada uno de ellos desde su lugar y tiempo, propuso soluciones para revertir, disminuir, controlar o eliminar los problemas asociados a los abusos, omisiones y errores que ya se han comentado.

Como estrategia de acción muchas de estas personas coincidieron en la idea de sustentabilidad e iniciaron con el uso de diversos términos relacionados a ella. De esta manera desde diferentes circunstancias históricas y marcos de referencia, se construyó el significado de las palabras "sostenible", "sustentable", "sostenido", "sustentabilidad" y "sostenibilidad". Sus diversas interpretaciones produjeron una especie de trayectoria que el presente trabajo busca recorrer.

Es importante mencionar que no se trata de mostrar un enfoque gramatical, sino de analizar el significado que contienen en su expresión tales términos, ya que se parte del hecho de que 
antes de la expresión, el ser humano construyó un modelo que codificó las propiedades o significados de la palabra que empleará (Gutiérrez-Rexach, 2016).

La importancia de este análisis radica en aclarar que, con el tiempo, el conjunto de interpretaciones se convirtió en una dispersión conceptual que hoy en día dificulta el cumplir acuerdos para enfrentar los diversos problemas socio-ecosistémicos. Por ejemplo, una empresa refresquera podría colocar en su discurso que es sustentable porque siembra árboles, mientras que el gobierno local le explica que sus descargas de agua residual y su explotación del acuífero se acercan a la insustentabilidad.

Así que, para interpretar el significado que los seres humanos hemos colocado en esas palabras resulta fundamental reconocer la intencionalidad con la cual se han utilizado. Para lograr lo anterior, se hará uso de la siguiente clasificación (Tabla 1) a efecto de identificar el propósito, la centralidad, y la importancia que se le da a los recursos naturales, en cada tipo de semántica.

Tanto en la no conservacionista como en la de optimización, el ser humano se ubica en el centro del discurso (antropocentrismo); cabe hacer notar que en muchos casos esa centralidad se llega a ubicar solo en un fragmento del mundo humano: la economía, dando origen a un econocentrismo.

Por su parte, para la semántica conservacionista los ecosistemas representan el eje principal de interés.

Tabla 1. Clasificación de las perspectivas en el empleo de los términos asociados a la sustentabilidad. Fuente: elaboración propia a partir de Foladori \& Pierri (2005), Gallopín (2003) y Gil Corrales (2007).

\begin{tabular}{|c|c|c|c|}
\hline & $\begin{array}{l}\text { PROPÓSITO } \\
\text { PRINCIPAL }\end{array}$ & CENTRALIDAD & $\begin{array}{l}\text { IMPORTANCIA DE LOS } \\
\text { RECURSOS NATURALES }\end{array}$ \\
\hline $\begin{array}{l}\text { Semántica NO } \\
\text { CONSERVACIONISTA }\end{array}$ & $\begin{array}{l}\text { Satisfacer las } \\
\text { necesidades } \\
\text { humanas infinitas. }\end{array}$ & Antropocéntrica. & $\begin{array}{l}\text { Se omite el hecho de que los } \\
\text { recursos naturales son } \\
\text { limitados. }\end{array}$ \\
\hline $\begin{array}{l}\text { Semántica de } \\
\text { OPTIMIZACIÓN }\end{array}$ & $\begin{array}{l}\text { Satisfacer las } \\
\text { necesidades } \\
\text { humanas que el } \\
\text { mercado } \\
\text { disponga. }\end{array}$ & Antropocéntrica. & $\begin{array}{l}\text { Acepta que los recursos } \\
\text { naturales son limitados y busca } \\
\text { hacer eficiente su uso a través } \\
\text { de estrategias de optimización } \\
\text { - multiplicación del recurso, } \\
\text { mejora en su manejo y } \\
\text { distribución, cambio de valor } \\
\text { en el mercado, incremento en } \\
\text { los rendimientos por medios } \\
\text { científicos y tecnológicos-. }\end{array}$ \\
\hline $\begin{array}{l}\text { Semántica } \\
\text { CONSERVACIONISTA }\end{array}$ & $\begin{array}{l}\text { Satisfacer las } \\
\text { necesidades } \\
\text { humanas finitas, } \\
\text { moderando la } \\
\text { cantidad y } \\
\text { modalidades de } \\
\text { consumo. }\end{array}$ & Ecocéntrica. & $\begin{array}{l}\text { Antepone la conservación de } \\
\text { los recursos naturales por su } \\
\text { valor inherente en sustentar la } \\
\text { vida y busca respetar una tasa } \\
\text { de equilibrio entre los procesos } \\
\text { antrópicos - extracción y } \\
\text { contaminación-y la } \\
\text { capacidad de recuperación } \\
\text { natural del entorno. }\end{array}$ \\
\hline
\end{tabular}




\section{Incorporación de los términos asociados a la Sustentabilidad en el discurso humano}

\subsection{Hans Carl von Carlowitz y Sylvicultura oeconomica}

En la Europa del siglo XVIII, el administrador alemán de minería Hans Carl von Carlowitz publicó en 1713 el libro Sylvicultura oeconomica donde puso de manifiesto la gran devastación de los bosques europeos a manos de varias industrias: la relativa a la fundición minera, la correspondiente a la fabricación de barcos, y la agrícola (Carlowitz, 2012). Desde esa época von Carlowitz criticó el pensamiento cortoplacista contemporáneo, centrado únicamente en ganar dinero y ciego a los daños irreparables que se estaban produciendo en los bosques. En su obra identificó una serie de medidas prácticas que fueran capaces de lograr la conservación y el cultivo de la madera de forma continua, constante y sustentable. El término que usó fue nachhaltend o nachhaltig y constituye la primera referencia al término sustentable, aunque usado como adjetivo en el sentido de durable (Grober, 2007; Schmithusen, 2013). Es importante subrayar que la propuesta de von Carlowitz pertenece a la semántica conservacionista debido a que las medidas que propuso se originaron en la idea de que: (a) la Tierra no podría seguir existiendo sin bosques; (b) era necesario un equilibrio entre extracción y regeneración; (c) la economía no debería convertirse en deficitaria al arrancar en horas, lo que al bosque le había costado siglos (Huss \& Gadow, 2012).

Aunque el trabajo de von Carlowitz fue muy reconocido, la estructura económica de aquella época - el cameralismo alemán- continuó dando prioridad a una economía basada en la sustitución de importaciones, en la producción interna, y en la recaudación de impuestos y tributos por parte del estado absolutista administrador.

Posteriormente, en los siglos XIX y XX se publicaron muchos documentos donde se expusieron problemas ambientales derivados de vínculos económicos mal manejados en muchas partes del Planeta; aunque no hacían uso explícito de las palabras sustentable o sustentabilidad, si expresaban el sentido y significación de lo que representa el término. Debido a que el presente trabajo enfoca su atención en la trayectoria semántica al usar los términos, dichos trabajos no serán mencionados.

\subsection{Meadows y Los límites del Crecimiento}

Fue hasta el periodo de 1970 a 1972 que en el Massachusetts Institute of Technology (MIT), un equipo interdisciplinario de 16 investigadores dirigidos por Dennis Meadows abordó el estudio de un conjunto de problemas que preocupaban a todas las naciones, pero que eran de especial interés para el Club de Roma (Gardner, 2004). El proyecto consistió en analizar qué pasaría con el abastecimiento de recursos naturales si continuara el crecimiento ilimitado de la población, de la producción industrial, de la producción de alimentos, y de la contaminación. Es decir, cómo reaccionaría la naturaleza si los vínculos antrópicos crecieran sin ningún límite, dejando que la política económica del laissez-faire prevaleciera en todos los ámbitos.

Sus resultados fueron publicados bajo el título The Limits to Growth, y mostraban que si las tendencias de crecimiento de la población mundial, de la industrialización, de la contaminación, y de la producción de alimentos continuaran al mismo ritmo, los recursos se agotarían en algún momento de los próximos 100 años —a partir de 1972- debido a que se rebasarían los límites del crecimiento. Posteriormente ocurriría un declive bastante repentino e incontrolable tanto de la población como de la capacidad industrial (Meadows et al., 1972).

Concluyeron con esperanza que también era posible alterar las tendencias identificadas y establecer condiciones de estabilidad ecológica y económica que fueran sustentables en el 
futuro - la palabra empleada en el texto en inglés fue sustainable-; su uso refiere un adjetivo para las nuevas condiciones que podrían lograrse y que deberían situarse en un estado de equilibrio global de acuerdo con el número de habitantes, el consumo de recursos naturales y la menor producción industrial.

Esas nuevas condiciones abarcan diversas propuestas ${ }^{1}$, y constituyen en sí una iniciativa de semántica conservacionista, ya que identifica muy bien el papel de soporte vital que la naturaleza tiene y, además, que la única forma de cuidarla es disminuyendo producción, consumo y población que se lleva a cabo en la Antropósfera² ${ }^{2}$.

\subsection{A Blueprint for Survival}

Las ideas vertidas en The Limits of Growth fueron respaldadas y reforzadas por un grupo interdisciplinario de 36 investigadores involucrados en el análisis de problemas ambientales globales a través del texto denominado A Blueprint for Survival. La publicación fue realizada en 1972 y en ella se utiliza con más familiaridad el término sustentable — la palabra empleada en el texto en inglés fue sustainable- Su empleo se realiza como adjetivo que aplica a la sociedad y su significado apunta hacia el hecho de que es la sociedad la que debe adaptarse a nuevas condiciones que logren la estabilidad y bienestar de la naturaleza, y con ello la estabilidad de la sociedad.

De forma específica, A Blueprint for Survival propuso una mínima alteración de los procesos ecológicos, una conservación máxima de materiales y energía mediante una economía de stock y no de flujos, un control de la población donde el "reclutamiento" igualara a las pérdidas, y un sistema social en el que todos los individuos disfrutaran y participaran de las tres condiciones anteriores en lugar de sentirse limitados por ellas (Goldsmith et al., 1972). El cumplimiento de todo lo anterior debería traer como consecuencia que las sociedades se sustentaran indefinidamente — sociedad estable — otorgando una satisfacción óptima a sus individuos.

Se puede señalar que esta propuesta colectiva interpreta bien el valor inherente de la naturaleza y busca la disminución en la producción y consumos humanos, por lo que constituye un ejemplo de semántica conservacionista más estricta.

\subsection{Declaración de Estocolmo}

En 1972 se llevó a cabo la Conferencia de las Naciones Unidas sobre el Medio Humano en Estocolmo. Su relevancia no radica en la integración de la palabra sustentable o sustentabilidad al discurso de la Organización de las Naciones Unidas (ONU), sino en la incorporación a la agenda política y económica mundial de la necesidad de proteger y mejorar el "medio humano"3 (Asamblea General de las Naciones Unidas, 1972). Puede decirse que los Principios de esta Declaración jugaron un papel fundamental para establecer y formalizar la

\footnotetext{
${ }^{1}$ (a) Estabilización de la tasa de natalidad igual a la de mortalidad en 1975; (b) Dejar que el capital industrial aumente naturalmente hasta 1990, luego de lo cual se estabiliza fijando tasas de inversión igual a tasas de depreciación; (c) Reducir el consumo de recursos naturales no renovables a una cuarta parte del valor alcanzado en 1970; (d) Reorientar las preferencias económicas de la sociedad hacia los servicios (educación y sanidad) y menos hacia los bienes materiales producidos en fábricas; (e) Reducir a una cuarta parte la generación de contaminación (industrial y agrícola) respecto a 1970; (f) Desviación del capital a la producción de alimentos aunque esta inversión fuera considerada "antieconómica" Meadows et al. (1972).

2 Esta palabra hace alusión a toda la población humana, sus artefactos, tecnología, ciencia, cultura, urbanizaciones, zonas agrícolas y ganaderas y las demás donde ejerce control y poder Manahan (2000).

${ }^{3}$ Referido en el texto como aquel que incluía tanto al entorno artificial como al natural.
} 
existencia de un vínculo de obligación y responsabilidad que los seres humanos adquirimos cuando ejercemos los derechos de uso sobre los recursos naturales. Es decir, ya no solo se validaba el vínculo económico, sino que se abogaba por un nuevo vínculo de obligación con la naturaleza.

A lo anterior hay que adicionar que en la Declaración de Estocolmo la sostenibilidad figura sin figurar debido a que integra al marco político internacional, que antes solo había considerado cuestiones económicas y sociales, el vínculo entre el desarrollo humano y la naturaleza (Du Pisani, 2006). Además, el trabajo de esta Conferencia resultó sumamente relevante porque condujo a la creación del Programa de las Naciones Unidas para el Medio Ambiente (PNUMA) y, con ello, a la construcción de un portal de acceso para las palabras "sostenible", "sustentable", "sostenido" y "sustentabilidad", y su significado en la agenda internacional.

\subsection{Informe Brundtland}

En 1984 la ONU creó la Comisión Mundial sobre el Medio Ambiente y el Desarrollo con la finalidad de ser un organismo rector de las cuestiones del medio ambiente en todo el mundo. Con un poco de tiempo y muchos antecedentes, esta Comisión conjuntó en el discurso internacional a dos elementos clave y al parecer contradictorios, el desarrollo y el medio ambiente. La Comisión ha explicado que limitar sus atribuciones a cuestiones medioambientales hubiera sido un error y una ingenuidad, ya que el medio ambiente no podría existir separado de las acciones, las ambiciones y las necesidades humanas; de forma textual expone que "el medio ambiente es donde vivimos todos, y el desarrollo es lo que todos hacemos al tratar de mejorar nuestra suerte en el entorno en que vivimos, y ambas son inseparables" (Asamblea General de las Naciones Unidas, 1987).

Con esta combinación de enfoques, la Comisión preparó por tres años un documento para la Asamblea General, presentando así en 1987 el Informe de la Comisión Mundial sobre el Medio Ambiente y el Desarrollo, más conocido como Nuestro Futuro Común o Informe Brundtland.

El reto planteado a la Comisión era presentar al mundo la posibilidad de una nueva era de crecimiento económico, sostenible social y medioambientalmente a largo plazo que fuera una realidad al año 2000 y más allá. La propuesta presentada fue designada como desarrollo sostenido en algunas partes del documento, en otras como desarrollo duradero y solamente en una ocasión se hace alusión a que "el desarrollo sea sostenible" — de acuerdo con al documento original de las Naciones Unidas publicado en español- (Asamblea General de las Naciones Unidas, 1987).

El resultado de la fusión conceptual concluyó que el futuro común de los pueblos del mundo obligaba a la inmediata cooperación internacional más allá de fronteras y de ganancias económicas a efecto de: “(a) crear políticas que sostengan y amplíen los recursos de la base Tierra; (b) no poner en peligro los sistemas naturales; (c) crear un desarrollo económico que se base sólidamente en las existencias reales de capital que lo sostiene; y (d) asegurar que las tasas de explotación de los recursos renovables se mantengan dentro de los límites de rendimientos sostenibles y que se disponga de fondos para regenerar los recursos y encarar todos los efectos vinculados con el medio ambiente" (Asamblea General de las Naciones Unidas, 1987). 
El Informe Brundtland representa una semántica de optimización cuya intención fundamental es conservar y mantener el progreso humano, asegurando que se satisfagan las necesidades del presente sin comprometer la capacidad de las futuras generaciones para satisfacer las propias. Las palabras "sostenible" y "sostenido" se usan como adjetivos para el desarrollo e indican que el capital natural debe optimizarse para que el stock no disminuya. No se plantea en ningún punto la disminución de los consumos humanos o el control de las ambiciones, como estrategia para el desarrollo sostenido.

El contenido de este informe muestra, por un lado, que los problemas medioambientales ya se habían integrado al discurso y agenda política internacional y, por el otro, que seguía permeando la naturaleza antropocéntrica en la propuesta.

\section{La dispersión semántica en el discurso}

En los tiempos posteriores al Informe Brundtland los adjetivos "sustentable", "sostenido" y "sostenible", multiplicaron su uso y polinizaron semánticamente a muchos sustantivos en contextos diferentes a los del desarrollo y sociedad.

Dicha dispersión lingüística produjo un nuevo espacio semántico donde los diferentes ámbitos académicos, políticos, sociales y económicos buscaban dar su propia interpretación a dichas palabras. En esa oportunidad, iniciada aproximadamente en los años noventa del siglo pasado, los seres humanos nos vimos en la necesidad de comprender la diferencia entre esos nuevos términos y particularmente en el idioma español apareció una pregunta que a lo largo del tiempo se ha realizado a diferentes especialistas, en diversos países ¿es lo mismo sostenible que sustentable?

Para dar respuesta al cuestionamiento anterior y, además, para decidir cómo emplear las nuevas palabras, se produjeron - y aún hoy siguen generándose - una heterogeneidad de interpretaciones que pueden agruparse en tres sentidos.

A. La primera de ellas consiste en negar la sinonimia y diferenciar la característica que cada adjetivo le daría al sustantivo. El trabajo realizado para tal fin por la Real Academia Española (RAE) expone esta posición al indicar que:

- Sostenible es un "adjetivo que significa que se puede sostener" (acepción 1/e.g. opinión, situación sostenible) o bien un "adjetivo usado especialmente en ecología y economía, que se puede mantener durante largo tiempo sin agotar los recursos o causar grave daño al medio ambiente" (acepción 2/e.g. desarrollo, economía sostenible) (Real Academia Española, 2021). Ésta última sería una interpretación de utilidad para la narrativa expuesta en este artículo.

- Sustentable es un "adjetivo que significa que se puede sustentar o defender con razones” (Real Academia Española, 2021). Desde esta perspectiva esta acepción no resultaría útil para el contexto de lo que se ha planteado a lo largo de este artículo.

B. El segundo sentido para los términos fue considerarlos sinónimos entre sí y colocarlos como adjetivos a un sustantivo al cual convendría mantener por un tiempo prolongado. Este sustantivo podría ser Desarrollo, Crecimiento, Sociedad, Arquitectura, Tecnología, Agricultura, Economía, Empresa, Campus Universitario, Producción, Consumo, Construcción y Ecosistema, entre muchos otros. Es importante destacar que para cada caso 
el centro de atención es el sustantivo y, por tanto, es a él a quien se pretende sostener, ignorando por completo que para que pueda seguir desarrollándose tal o cual actividad, lo que debe subsistir es el entorno natural sobre el que se desplanta o que le da origen. Este empleo de los términos no responde al significado expuesto por la RAE con respecto a ser una actividad que se puede mantener durante largo tiempo sin agotar los recursos o causar grave daño al medio ambiente.

Otro aspecto que hay que destacar en esta segunda categoría, es la decisión de ciertos países e instituciones de adoptar solo a una de estas palabras, evitando a las otras como una estrategia para prevenir la confusión, pero a la vez, sin el interés de profundizar en los conceptos. $^{4}$

Invariablemente como adjetivo, a la palabra se le dotó de una capacidad de mejoramiento ecológico y de reductor de problemas ambientales, pues se ha interpretado que el hecho de adicionarla es una señal inequívoca de resolver los conflictos ambientales; en consecuencia, se ha empleado con fuerza aún en la semántica no conservacionista. Un ejemplo de lo anterior es la integración de dos términos más opuestos que similares: la "industria" y "sustentable". Es el caso de la estrategia para el Desarrollo Industrial Sustentable e Inclusivo (ISID) desarrollada por la Organización de las Naciones Unidas para el Desarrollo Industrial (ONUDI), cuyas metas son "crear prosperidad compartida gracias al trabajo que las industrias proveen-, y salvaguardar el medio ambiente mediante una producción más limpia, una gestión eficiente de los recursos y una reducción de los residuos y de la contaminación- (United Nations Industrial Development Organization, 2014). Cabe considerar que en ningún momento se contempla la existencia de límites que enfrenten los verdaderos problemas que las industrias representan: sobreexplotación de los recursos naturales, sobreproducción de bienes humanos, y la alta emisión de GEI asociada a ellas.

C. La tercera categoría corresponde a la conversión de adjetivo a sustantivo para dar origen a las palabras "sustentabilidad" y "sostenibilidad". Se trata de una postura académica que al comprender la confusión que se había originado con la adjetivación, decidió establecer esta alternativa: usar los nuevos sustantivos para indicar el sostenimiento en el tiempo de las condiciones naturales que nutren y mantienen a las actividades antrópicas. De esta manera se estableció la clasificación de la sustentabilidad en muy fuerte, fuerte, débil y muy débil, como una escala basada en la sustituibilidad entre el capital natural y el manufacturado (Foladori \& Pierri, 2005). Es decir, considerar que la madera de un bosque es un bien natural capaz de ser sustituido por rollos de papel bond, es un ejemplo de sustentabilidad muy débil.

Incluso desde la academia, en otra propuesta, estos sustantivos fueron disgregados en tres sectores - social, económico y ambiental - para dar origen al famoso esquema de esferas en interacción. Posteriormente a esta representación se le añadieron los ámbitos tecnológico y ético con la intención de completar dos aspectos muy relevantes en su consecución (Schmitt Siqueira Garcia \& Siqueira Garcia, 2020).

\footnotetext{
${ }^{4}$ Por ejemplo, para la Organización de las Naciones Unidas se emplea el término sostenible en la traducción al español (e.g. Objetivos de Desarrollo Sostenible); y en la península ibérica también se prefiere la utilización de sostenible, en concordancia con la RAE.
} 
Resulta importante resaltar que, en este orden de ideas, ambos sustantivos fueron considerados nuevamente como sinónimos y empleados de forma más frecuente en unos países que en otros.

No obstante, frente a estas nuevas palabras, la RAE — además de seguir estableciendo la ausencia de sinonimia-, describió a la sustentabilidad como "una cualidad de sustentable", y a la sostenibilidad como "una cualidad de sostenible" (Real Academia Española, 2021). Eso significa la no aceptación como sustantivos, sino la vuelta a colocarlos como adjetivos o "cualidad de". Desde esta perspectiva sería lo mismo decir arquitectura sostenible o sostenibilidad de la arquitectura. En definitiva, representa un retroceso a la iniciativa académica inicial de aclarar los términos y de incluir la importancia del sostenimiento en el tiempo de las condiciones naturales que nutren y mantienen a las actividades antrópicas.

\section{Reflexiones finales}

En el desarrollo del presente trabajo se describieron las múltiples variaciones en el uso e interpretación de los términos "sustentable", "sostenido", "sostenible", "sustentabilidad" y "sostenibilidad", y quedó de manifiesto que la amplia permisividad en el uso y significado de las palabras ha fomentado la actual polisemia de los términos.

Lo anterior ha promovido que se produzcan constantemente confusiones conceptuales y se hagan constantemente preguntas como ¿qué se está sustentando? — como si cualquier asunto debiera perdurar o sustentarse, aunque muestre altos costos para el entorno natural一, o bien, ¿qué diferencia existe entre sustentable y sostenible? - como si lo relevante para enfrentar los problemas que afrontamos como seres humanos en el siglo XXI dependieran del uso de una u otra palabra-.

Y aunque es cierto que la multiplicidad de interpretaciones intensificó la disección del significado, es momento de analizar todo lo aprendido en el camino polisémico narrado anteriormente. Fue natural que, al tratarse de nuevos términos, la naturaleza humana permitiera a estas palabras atravesar una etapa nómada desde un nicho semántico al otro. Pero hoy resulta pertinente y urgente impulsar su ubicación en un solo principio explicativo.

Sean estas reflexiones finales la invitación a coincidir en una visión convergente en el uso de los términos "sustentable", "sostenido", "sostenible", "sustentabilidad" y "sostenibilidad". De forma concisa: su empleo debería coincidir con la idea de mantener las condiciones de la atmósfera, la hidrósfera, la litósfera y la biósfera en el estado del Holoceno, ya que éstas han permitido salvaguardar al entorno natural que nutre, conserva y protege a nuestra especie. Tales condiciones son las que deberían ser valiosas para el ser humano porque representan el sustrato sobre el que se desplanta, y destruirlo lo aniquila así mismo.

Considérese que, si se lograran sustentar las condiciones del entorno natural y adaptar a la sociedad y a la economía a ellas, entonces por consecuencia se lograrán las tan ansiadas sociedades y economías sustentables.

Todo lo anterior adquiere relevancia porque la flexibilidad en los significados ha obstaculizado el avance óptimo y eficiente para enfrentar la crisis civilizatoria que se sintetiza en el actual Cambio Climático en el que todos estamos inmersos. El impacto más significativo 
deriva del hecho que al permitir que cada actor social, político y económico interprete bajo su criterio, se propicia la construcción de múltiples vías dispersas y divergentes que evitan la conjunción de esfuerzos globales. Como se ejemplificó antes, una empresa refresquera podría colocar en su discurso que es sustentable porque siembra árboles, mientras que el gobierno local la considera insustentable por la extracción excesiva que hace del agua de un acuífero y el vertido de aguas residuales sin tratar.

¿Qué hacer con la polisemia que tenemos entre manos? Hay que redireccionar su significado hacia la perspectiva conservacionista en la intención de sustentar las condiciones de habitabilidad humana en nuestro Planeta Tierra. Coloquemos las ideas vertidas en este trabajo sobre una mesa de análisis que detenga el nomadismo de los términos; es hora de que se alojen de forma definitiva en un sitio que homogenice su semántica en bien de la naturaleza y de los propios seres humanos.

\section{Referencias}

Abbot, G., Farndon, J., Kennedy, F., Meadway, J., Wallace, C., Weeks, M., \& Kishtainy, N. (2019). El Libro de la Economía (Cuarta reimpresión). Penguin Random House.

Asamblea General de las Naciones Unidas. (1972). Declaración de la Conferencia de las Naciones Unidas sobre el Medio Humano. Declaración de Estocolmo. SEMARNAT. https://biblioteca.semarnat.gob.mx/janium/recursos/224844/Contenido/B\%20declaraci ones/3\%20Declaracion\%20Medio\%20Humano.pdf

Asamblea General de las Naciones Unidas. (1987). Informe de la Comisión Mundial sobre el Medio Ambiente y el Desarrollo "Nuestro Futuro Común". Acta del cuadragésimo segundo periodo de sesiones. ONU.

Avio, C. G., Gorbi, S., \& Regoli, F. (2017). Plastics and microplastics in the oceans: From emerging pollutants to emerged threat. Marine Environmental Research, 128, 2-11. https://doi.org/10.1016/j.marenvres.2016.05.012

Carlowitz, H. C. von. (2012). Sylvicultura oeconomica: Hausswirthliche Nachricht und Naturmäßige Anweisung zur Wilden Baum-Zucht Facsímil de la primera edición (Facsímil de la primera edición de 1713). Verlag Kessel.

Cruz, P. M., Bodnar, Z., \& Staffen, M. R. (2020). Amazon in flames: for a multilevel environmental transnational governance. Sostenibilidad: económica, social $y$ ambiental, 2, 66-77. https://doi.org/10.14198/Sostenibilidad2020.2.05

Du Pisani, J. A. (2006). Sustainable development - historical roots of the concept. Environmental Sciences, 3(2), 83-96. https://doi.org/10.1080/15693430600688831

Environmental Justice Organisations, Liabilities and Trade. (revisado abril 2021). Mapa Mundial de Justicia Ambiental. https://ejatlas.org/?translate=es

Foladori, G., \& Pierri, N. (Eds.). (2005). ¿Sustentabilidad? Desacuerdos sobre el desarrollo sustentable (Primera edición). Miguel Ángel Porrúa. 
Gallopín, G. (2003). Sostenibilidad y desarrollo Sostenible: un enfoque sistémico. Serie Medio Ambiente y Desarrollo. División de Desarrollo Sostenible y Asentamientos Humanos de CEPAL.

Gardner, T. (2004). Limits to Growth? - A Perspective on the Perpetual Debate. Environmental Sciences, $1(2), \quad 121-138$. https://doi.org/10.1080/15693430512331342592

Gil Corrales, M. Á. (2007). Crónica Ambiental. Gestión Pública de Políticas Ambientales en México (Primera Edición). Fondo de Cultura Económica.

Goldsmith, E., Allen, R., Allaby, M., Davoll, J., \& Lawrence, S. (1972). A Blueprint for Survival. The Ecologist, 2(1), 1-22.

Grober, U. (2007). Deep roots: A conceptual history of "sustainable development" (Nachhaltigkeit). ECONSTOR. WZB Discussion Paper, No. P 2007-002, Wissenschaftszentrum Berlin für Sozialforschung (WZB), Berlin. https://www.econstor.eu/bitstream/10419/50254/1/535039824.pdf

Gutiérrez-Rexach, J. (Ed.). (2016). Enciclopedia de Lingüística Hipánica, Vol I: La Semántica (Volumen I). Routledge. https://doi.org/10.4324/9781315713441

Huss, J., \& Gadow, F. von. (2012). Hannß Carl von Carlowitz Sylvicultura oeconomica Hausswirthliche Nachricht und Naturmäßige Anweisung zur Wilden Baum-Zucht Faksimile der Erstauflage Leipzig 1713: Theoretische Grundlagen, Bildungskonzept für die Schulpraxis, Wirkungen; eine Fallstudie an der Grundschule Mohorn (Sachsen). Verlag Kessel.

Kraus, H.C. (2011). Del Cameralismo a la Economía Nacional. La tradición alemana de las ciencias políticas hasta el comienzo del siglo XX. Historia 396, 1, 103-125.

Manahan, E. S. (Ed.). (2000). The Anthrosphere, Industrial Ecosystems, and Environmental Chemistry. CRC Press LLC. http://www.ingenieroambiental.com/2020/1492ch02.pdf

Meadows, D [Donella], Meadows, D [Dennis], Randers, J., \& Behrens, W. (1972). The Limits to Growth. A Report for the Club of Rome's Project on the Predicament of Mankind. Potomac Associates Book. https://doi.org/10.1349/ddlp.1

Real Academia Española. (2021, junio en línea). Diccionario de la Lengua Española. https://www.rae.es/

Robbins, L. (1932). Ensayo sobre la naturaleza y significación de la ciencia económica (1944 al español). Fondo de Cultura Económica. https://www.ucursos.cl/iei/2012/4/MEIYPCO1/1/material_docente/bajar\%3Fid_material\%3D596941

Schmithusen, F. (2013). La sostenibilidad aplicada en el sector de las actividades forestales cumple 300 años. Unasylva, 64, 240, 1-9. 
Schmitt Siqueira Garcia, D., \& Siqueira Garcia, H. (2020). La dimensión ética de la sostenibilidad: la necesidad de cambiar valores y actitudes. Sostenibilidad: económica, social y ambiental, 2, 33-47. https://doi.org/10.14198/Sostenibilidad2020.2.03

United Nations Industrial Development Organization. (2014). Inclusive and Sustainable Industrial Development. ONU.

Williams, J. (2005). La desecación de un mar: de cómo el algodón dejó al Aral sin agua y sin gente. Ecología Política, 33, 89-92.

Yu, P., Xu, R., Abramson, M. J., Li, S., \& Guo, Y. (2020). Bushfires in Australia: a serious health emergency under climate change. The Lancet Planetary Health, 4(1), e7-e8. https://doi.org/10.1016/S2542-5196(19)30267-0 